\title{
Management of patients with non-invasive positive pressure ventilation therapy in intensive care units
}

Nermin Olgun, RN; PhD, Associate Professor, College of Nursing, Medical Nursing Department, Marmara University, Istanbul, Turkey Halise Taskin, RN; MS, Staff Nurse, Intensive Care Unit, Marmara University Hospital, Istanbul, Turkey

E-mail: nerminolgun@superonline.com

Key words: Continuous positive airway pressure intensive care mask non-invasive ventilation pressure ulcer prevention and control

\section{SUMMARY \\ * Non-invasive positive pressure ventilation (NIPPV) refers to the provision of ventilatory support for a spontaneously breathing patient in the absence of endotracheal intubation. \\ * NIPPV with a facemask was first introduced in the early 1960s, because endotracheal intubation and ventilation, despite being effective and reliable, were associated with many complications. \\ * From the early 1980s onwards one of the main factors that contributed to the popularisation of NIPPV was the successful use of continuous positive airway pressure (CPAP) to treat obstructive sleep apnoea. \\ * CPAP provides continuous positive pressure without the need for mechanical support, and is administered to a patient during inspiration and expiration. \\ * Another type of non-invasive ventilation is bi-level positive airway pressure (BiBAP), whereby separate pressure adjustments can be made for inspiration and expiration.}

\section{INTRODUCTION}

Non-invasive positive pressure ventilation (NIPPV) provides assistance to a patient's respiration by delivering pressurised gas to the airway, which increases transpulmonary pressure and inflates the lungs. Exhalation is achieved via the elastic recoil of the lungs and sometimes by active contraction recoil of expiratory muscles. The main difference between invasive ventilation and NIPPV is that gas is given via a mask in NIPPV. Bi-level positive airway pressure (BiPAP) describes a form of NIPPV in which there are separate pressure adjustments for the inspiratory and expiratory phases. Continuous positive airway pressure (CPAP) is the non-invasive application of positive airway pressure through a mask without the provision of ventilatory support. It is therefore not considered to be a form of non-invasive ventilation, but serves to stimulate alveolar ventilation in a spontaneously breathing patient. Due to the similar nature of the associated issues, CPAP is often discussed simultaneously.

This article discusses the indications, applications and complications associated with NIPPV. In addition, an overview of the management of patients receiving this therapy is included.

\section{BACKGROUND}

NIPPV with a facemask was first introduced in the early 1960s, because endotracheal intubation and mechanical ventilation, despite being effective and reliable, were associated with many complications. However, underdeveloped mask technology at that time and inappropriate mask sizes limited the success of NIPPV. Thus, the use of invasive mechanical ventilation overtook the use of NIPPV. In 1984, French researchers proposed the use of mask ventilation for Duchenne's muscular dystrophy to decrease or eliminate muscle fatigue during the night. Shortly after, the success of nocturnal nasal ventilation in reducing gas exchange disorders and their symptoms was being reported for various neuro-muscular diseases and chest wall deformities. The last decade has seen a NIPPV revival, chiefly related to improvements in mask technology and ventilation systems.

NIPPV is now frequently the first line treatment in selected groups of patients who require respiratory support (BTS, 2002). Ideally NIPPV is applied to patients whose $\mathrm{pH}$ values are below 7.35 and above 7.45 , such as those with respiratory acidosis due to hypoxia with $\mathrm{CO}_{2}$ retention and those with respiratory alkalosis due to ineffective rapid and shallow breathing.

The rationale for initially using NIPPV on these patients is to avoid the invasive procedure of endotracheal intubation, decreasing the likelihood of infection and other complications associated with the use of an artificial airway. In addition, the use of NIPPV may prevent admission to an intensive care unit and shorten the hospitalisation period. It also has the added advantages of simplicity, maintenance of the patient's ability to communicate and swallow and the ease of implementation and discontinuation. For these reasons, the use of NIPPV is becoming more widespread.

However, NIPPV treatment may not be readily accepted by all patients. One of the complications of its use is the prevalence of facial and oral ulceration (Smith et al., 1998; Brigg, 1999; Marshall \& Pittard, 1999). For some patients it means admission to an intensive care unit which can lead to them experiencing increased levels of panic, anxiety and agitation due to the disturbing environment. In addition, as a consequence of the need for a tightly fitting face mask, this treatment is also difficult to accept for 
patients with claustrophobia.

\section{PHYSIOLOGICAL EFFECTS OF NIPPV}

The physiological effects of NIPPV include improved oxygenation, less effort needed to breathe, improved ventilation/perfusion (V/Q) matching, decreased fatigue and increased minute ventilation (Meduri, 1996; Hotchkiss \& Marini, 1998; Duke \& Bersten, 1999). The application of positive pressure to the airways increases alveolar distension, helps prevent alveolar collapse and, as a result, has the potential to increase the transfer of oxygen at the alveolar-capillary membrane. In patients with interstitial oedema, the mechanical effects of alveolar distension help the dispersion of interstitial fluid and potentially increase oxygen transfer to capillary blood. NIPPV may also be effective in providing rest for chronically fatigued muscles (Brochard et al., 1990). In studies of the haemodynamic effects of NIPPV, no significant differences were noted in the pulmonary arterial pressure or in cardiac output, although a significant reduction in pulse rate was identified (Çelikel, 2000; Koç \& Yüksel, 2003; Vanpee, 2003).

\section{NIPPV INDICATIONS}

Several randomised and non-randomised clinical trials have demonstrated that NIPPV is an effective treatment in the management of acute respiratory failure (Antonelli et al., 2002). The indications of its use can be summarised as:

* Chronic obstructive pulmonary disease (COPD) with acute respiratory acidosis;

* Long-term homecare of COPD patients;

* Obstructive sleep apnoea syndrome;

* Hypercapnic respiratory failure due to chest wall deformities or neuromuscular disorders;

* Cardiogenic pulmonary oedema (usually BiPAP and only when the condition remains unresponsive to CPAP);

* Acute pneumonia;

* Weaning from mechanical ventilation.

\section{COPD with respiratory acidosis}

NIPPV is increasingly the first choice treatment option for patients with exacerbated COPD and accompanying acidosis. A randomised controlled trial demonstrated a reduction in the number of intubations, improvements in dyspnoea scores and more stable vital signs in patients who received NIPPV. In addition, statistically significant differences were noted in mortality, morbidity and hospitalisation periods (Çelikel, 2000). Further studies have demonstrated the benefit of the early use of NIPPV in these patients (Brochard et al., 1995; Kramer, 1995 ; Bardi et al., 2000 ).

\section{Restrictive pulmonary disease}

The British Thoracic Society (BTS, 2002) recommends the use of NIPPV as the first-line treatment for patients with decompensated ventilatory failure due to neuromuscular disease or chest wall deformity. There is a lack of controlled studies in this area and the recommendation is based on their success of NIPPV with COPD patients.

\section{Hypoxaemic respiratory failure}

A randomised controlled trail conducted by Antonelli et al. (1998) compared NIPPV with endotracheal intubation and mechanical ventilation in 64 patients with hypoxaemic respiratory failure. The results demonstrated that NIPPV was just as effective as conventional ventilation in improving gas exchange, intubation was avoided in $69 \%$ of patients, and the incidence of sepsis and other complications was lower in the NIPPV group.

\section{Pulmonary oedema}

CPAP is effective in treating cardiogenic pulmonary oedema (Lin et al., 1995; Mehta et al., 1997). BiPAP may have a role to play if the patient's condition is unresponsive to CPAP (Murray, 2002).

\section{Immune-suppressive patients}

Due to the increased tendency to develop infection and more frequent incidences of haemorrhage with invasive mechanical ventilation, NIPPV has become the preferred method for use with immune-suppressive patients who have developed acute respiratory deficiencies (Koç \& Yüksel, 2003).

\section{Patients who are not suitable for intubation}

NIPPV may be applied to patients with malignancies that have a poor prognosis if they are intubated. It will assist in the reduction of dyspnoea and help them to maintain an acceptable quality of life.

\section{Weaning from mechanical ventilators and facilitating intubation \\ NIPPV may be used as a tool to support patients being weaned from mechanical ventilation. It has been shown to increase the likelihood of success when weaning, with a decrease in morbidity and mortality (Ferrer et al., 2003; Koç \& Yüksel, 2003). Nava et al. (1998) conducted a study comparing the use of standard weaning with the use of NIPPV. Participants were patients with COPD who had been intubated and ventilated, and who had experienced a failed weaning trial using a T-piece. The group who received NIPPV had reduced ventilation time (quicker weaning) and a reduction in the total number of intensive care days. The 60-day survival rate was $93 \%$ for the NIPPV group and $72 \%$ for the stan- dard weaning group.}

\section{Asthma}

Randomised controlled studies have been performed to examine the use of NIPPV in patients with asthma and cystic fibrosis. However, no conclusive results have been reported so far (Çelikel, 2000; Kaçmaz, 2002).

\section{COMPLICATIONS OF NIPPV}

There are a number of complications associated with the use of NIPPV. The most common ones are linked to masks, airflow and applied positive pressure.

\section{Complications related to masks}

Discomfort, irritation in the facial skin, claustrophobia, ulceration (see photo 1) at the nasal bridge (Meduri, 1996) and facial ulceration (Hill, 1993) are commonly seen in patients receiving NIPPV. In such cases, the mask strap may need to be loosened, and artificial support should be placed between the mask and nose or face. A study conducted by Callaghan and Trapp (1998) showed that the application of a Granuflex (ConvaTec Ltd) dressing on the nasal bridge reduces the pressure damage and may minimise air leaks. 


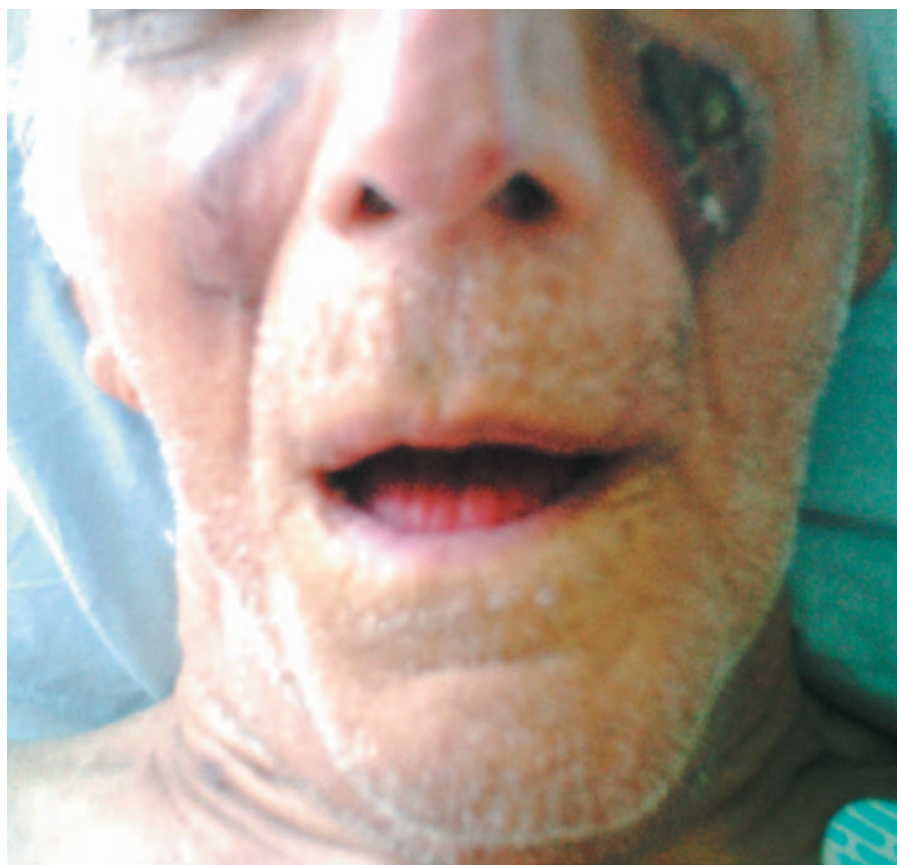

Photo 1: Face ulceration caused by CPAP mask pressure

\section{Complications related to airflow and pressure}

Nasal congestion, sinus/ear pain (Hotchkiss \& Marini, 1998), nasal/oral dehydration (Hayes et al., 1995), increased intraocular pressure (Alvarez-Sala et al., 1992), conjunctivitis (Hotchkiss \& Marini, 1998), gastric distension (Meduri, 1996) can all occur as a result of high pressure. Complications appear to be reduced when pressures of less than $25 \mathrm{~cm} \mathrm{H}_{2} \mathrm{O}$ are applied. Facial ulcerations are often related to ill-fitting masks or incorrect placement and irregular facial and oral care.

Major complications can be minimised with proper patient selection and preparation. Patients must be conscious and should be able to respond to verbal stimuli. Vomiting, aspiration and barotrauma appear to be rare complications of NIPPV (Hotchkiss \& Marini, 1998).

\section{CONTRAINDICATIONS TO THE USE OF NIPPV}

* Respiratory arrest - NIPPV is dependent on spontaneous respiratory effort;

* Cardiovascular instability - this is a controversial point. Confalonieri et al. (1994) indicate that the precise definition of instability is lacking in reported studies. Clinical judgement is required based on an individual needs assessment of the patient;

* Mental status derangement - co-operation of the patient influences the success and outcome of NIPPV. Patients who are confused or who have a lowered conscious level may not be able to tolerate this intervention;

* Excessive secretions or vomiting - due to the need to repeatedly remove the face mask, the benefits of NIPPV may not be achieved;

* Facial trauma or surgery - the application of a suitable face mask will be difficult;

* Recent oesophageal or gastric surgery - pressure in the upper airways may cause pressure on an anastomosis.

\section{OVERVIEW OF THE MANAGEMENT OF PATIENTS RECEIVING NIPPV}

The correct application and maintenance of masks are major factors in the success of NIPPV. Masks should suit the patients' facial anatomy. Although adaptation to this therapy is better with nasal masks, the use of face masks that cover the nose and mouth and the use of nasal buffers have been reported to be more effectivein reducing $\mathrm{CO}_{2}$ (Çelikel, 2000). Also, nasal masks appear to be tolerated more readily by patients who have claustrophobia.

The mask should be held in position using restraining straps to ensure a tight seal is obtained (see photo 2). A mask that is too loose will allow air leaks and one that is too tight may damage the nasal bridge. Attention to oral, facial and eye hygiene is crucial for maintaining the integration of facial tissues and mucosa. Oxygen should be administered and titrated to achieve a $\mathrm{SpO}_{2}$ greater than $90 \%$.

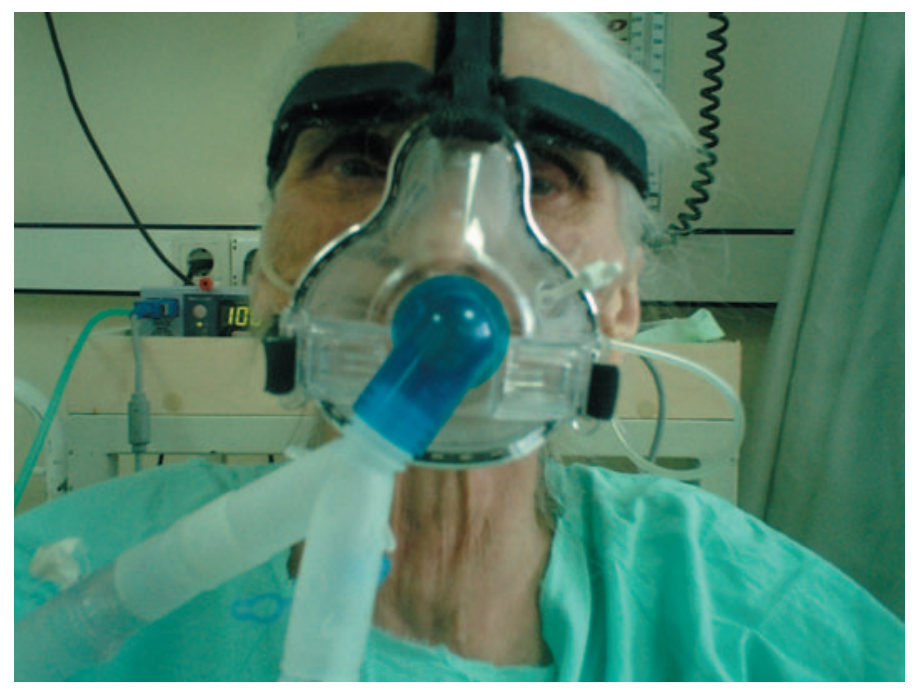

Photo 2: CPAP mask in place

When beginning the therapy, the mask should initially be applied using handheld pressure and the pressure levels increased slowly. The inspiratory level should be increased to one that the patient can tolerate. As the patient's condition begins to stabilise, the mask can be removed for short periods to allow oral intake.

The patient and the family need to be fully informed about the intervention and what is required of them. Patients will need to cooperate with medical and nursing staff, and the family can offer much-needed support and encouragement. Familiarisation with the equipment is important for initiating the therapy; the patient should be shown the equipment and agreement reached about methods of communication. The restraining straps generally have a quick release mechanism that the patient should be made aware of and its use demonstrated.

Cardiovascular and respiratory monitoring is required. Regular arterial blood gases are used to assess the effectiveness of the therapy. Pulse oximetry monitoring will allow continuous assessment of oxygenation. Continuous electrocardiogram (ECG) and frequent recording of blood pressure are advocated, particularly in the acute stages. The frequency can be reduced once the patient has stabilised. Assessment of the patient's respiratory rate and pattern will give an indication of how the patient is responding to the therapy. A reduction in respiratory rate and use of accessory muscles is expected as the patient's condition improves. 
The incidence of gastric distension is reported as being less than 2\% (Meduri, 1996; Duke \& Bersten, 1999) so the routine placement of a nasogastric tube is not required. If patients have, or subsequently develop, gastric distension a nasogastric tube is indicated.

\section{CONCLUSION}

Invasive mechanical ventilation is associated with a number of minor and major complications. NIPPV decreases the need for intubation and ventilation, and patients remain in control of their treatment and can communicate with the healthcare team. The benefits of NIPPV are evident from the literature; a key factor in its success is its early use.

\section{REFERENCES}

Alvarez-Sala R, Diaz S, Prados C, Villasante C, Villamor J. (1992) Increase in intraocular pressure using nasal CPAP (letter). Chest 101 (5), 1477.

Antonelli M, Conti G, Rocco M, Bufi M, De Blasi RA, Vivino G,

Gasparetto A, Meduri GU. (1998) A comparison of noninvasive positive-pressure ventilation and conventional mechanical ventilation in patients with acute respiratory failure. New England Journal of Medicine. 339, 429 - 435

Antonelli M, Mercurio G \& Pennisi M. (2002) Non-invasive mechanical ventilation and prevention of pneumonia in patients with acute respiratory failure. In: Vincent JL (ed). Yearbook of Intensive Care and Emergency Medicine. New York: Springer, pp352-361.

Bardi G, Pierotello R, Desideri M, Valdisseri L, Bottai M \& Palla A. (2000) Nasal ventilation in COPD exacerbations: early and late results of a prospective, controlled study. European Respiratory Journal. 15, $98-104$

Brigg C. (1999) The benefits of non-invasive ventilation and CPAP therapy. British Journal of Nursing 8 (20), 1355-1361.

Brochard L, Isabey D, Piquet J, Amaro P, Mancebo J, Messadi AA, Brun-Buisson C, Rauss A, Lemaire F \& Harf A. (1990) Reversal of acute exacerbations of chronic obstructive pulmonary disease by inspiratory assistance with face mask. New England Journal of Medicine 323 (22), 1523-1530.

Brochard L, Mancebo J, Wysocki M, Lofaso F, Conti G, Rauss , Simonneau G, Benito S, Gasparetto A, Lemaire F. et al (1995) Non-invasive ventilation for acute exacerbations of chronic pulmonary disease. New England Journal of Medicine 333, 817-822.

BTS (British Thoracic Society Standards of Care Committee). (2002) Non-invasive ventilation in acute respiratory failure. Thorax 57 (3), 192-211.

Callaghan S \& Trapp M. (1998) Evaluating two dressings for the prevention of nasal bridge pressure sores. Professional Nurse 13 (6), 361-364.

Çelikel T. (2000) Chronic Obstructive Pulmonary Diseases. Thorax Books. (1st Press, Volume 2) Istanbul: Thorax Association, pp137-157.

Confalonieri M, Aiolfi S, Scartabellati A. (1994) Non-invasive ventilation (letter). Annals of Internal Medicine 121 (12), 984-985.

Duke GJ \& Bersten AD. (1999) Non-invasive ventilation for adult acute respiratory failure. Part 1. Critical Care Resuscitation 1, 187-198.

Ferrer M, Esquinas A, Arancibia F, Bauer TT, Gonzalez G, Carrillo A, Rodriguez-Roisin R, Torres A. (2003) Non-invasive ventilation during persistent weaning failure: a randomized controlled trial. American Journal of Respiratory \& Critical Care Medicine. 168 (1), 70-76.

Hayes MJ, McGregor FB, Roberts DN, Schroter RC \& Pride NB. (1995) Continuous nasal positive airway pressure with a mouth leak: effect on nasal mucosal blood flux and nasal geometry. Thorax 50 (11), 1179-1182.

Hill NS. (1993) Non-invasive ventilation: does it work, for whom, and how? American Review of Respiratory Disease 147 (4), 1050-1055.

Hotchkiss JR \& Marini JJ. (1998) Non-invasive ventilation. An emerging supportive technique for the emergency department. Annals of Emergency Medicine 32 (4), 470-479.

Kaçmaz N. (2002) Patients of critical care that have psychological problems and nursing intervention. Journal of the Critical Care Nurses Society - Turkey 6 (2), 75-81.

Kramer N, Meyer TJ, Mecharg J, Cece RD, Hill NS (1995) Randomised prospective trial of non-invasive positive pressure ventilation in acute respiratory failure. American Journal of Respiratory Critical Care Medicine. 151, $1799-1806$

Koç M \& Yüksel E. (2003) Non-invasive mechanical ventilation in patients with COPD, In: Ozyardımcı N (ed). Thorax Diseases Clinical Seminary Study Book. Bursa: Uludag University Press.

Lin M, Yang YF, Chiang HT, Chang MS, Chiang BN \& Cheitlin MD. (1995) Reappraisal of continuous positive airway pressure therapy in acute cardiogenic pulmonary oedema. Short-term and long-term follow-up. Chest 107 (5), 1379-1386.

Marshall A \& Pittard M. (1999) Use of continuous positive airway pressure (CPAP) in the critically ill - physiological principles. Australian Critical Care 12 (4), 154-158.

Meduri GU. (1996) Non-invasive positive pressure ventilation in patients with chronic obstructive lung disease and acute respiratory failure. Current Opinion in Critical Care 2, 35-46.

Mehta S, Jay GD, Woolard RH, Hipona RA, Connolly EM, Cimini DM, Drinkwine JH \& Hill NS. (1997) Randomized, prospective trial of bi-level versus continuous positive airway pressure in acute pulmonary oedema. Critical Care Medicine 25 (4), 620-268.

Murray S. (2002) Bi-level positive airway pressure (BiPAP) and acute cardiogenic pulmonary oedema (ACPO) in the emergency department. Australian Critical Care 15 (2), 51-63.

Nava S, Ambrosino N, Clini E, Prato M, Orlando G, Vitacca M, Brigada P, Fracchia C \& Rubini F. (1998) Non-invasive mechanical ventilation in the weaning of patients with respiratory failure due to chronic obstructive pulmonary disease. A randomized, controlled trial. Annals of Internal Medicine 128 (9), 721-728.

Smith CE, Mayer LS \& Metsker C, Voelker M, Baldwin S, Whitman RA \& Pingleton SK. (1998) Continuous positive airway pressure: patients' and caregivers' learning needs and barriers to use. Heart \& Lung: The Journal of Acute and Critical Care 27 (2), 99-108.

Vanpee D. (2003) The use of non-invasive ventilation in the emergency department (editorial). European Journal of Emergency Medicine 10 (2), 77-78. 